\title{
Rapid progression of glioblastoma multiforme: A case report
}

\author{
YAN YAN ZHANG ${ }^{1}$, LING XIANG RUAN ${ }^{2}$ and SHENG ZHANG $^{2}$ \\ Departments of ${ }^{1}$ Neurology and ${ }^{2}$ Radiology, The First Affiliated Hospital, College of Medicine, \\ Zhejiang University, Hangzhou, Zhejiang 310003, P.R. China
}

Received April 13, 2015; Accepted August 10, 2016

DOI: $10.3892 / \mathrm{ol} .2016 .5228$

\begin{abstract}
Glioblastoma multiforme (GBM) tumors are intracranial lesions with varying shapes that grow rapidly. GBM tumors most commonly present as solitary lesions and multiple lesions are rare. The aim of the present case report was to investigate the imaging features of glioblastoma multiforme (GBM). In this study, the case of a 60-year-old patient who was hospitalized due to seizures is presented. Magnetic resonance imaging (MRI) revealed multiple lesions, heterogeneous in size, with peritumoral edema and ring-shaped enhancement. The lesions grew rapidly within 10 days of hospitalization and were initially misdiagnosed as either infections or intracranial metastatic tumors as a result of imaging examinations. The patient was subsequently administered mannitol, diazepam, Tegretol and ceftriaxone. After treatment, the patient recovered and regained full consciousness. However, MRI examination 23 days after hospitalization revealed that the multiple lesions in the left temporal and left occipital lobes had increased in size. Therefore, resection of the tumor in the left temporal occipital lobe was performed. Histopathological examination identified GBM (grade IV) in the left temporal and parietal lobes. The patient succumbed to the disease 7 months after surgery due to GBM recurrence. The findings of the present case indicate that GBM may progress rapidly with a doubling time of 10 days and multiple cystic alterations. Furthermore, if diagnosis of GBM is unclear, early biopsy is recommended.
\end{abstract}

\section{Introduction}

Glioblastoma multiforme (GBM) is an epithelial tumor of the central nervous system, which most commonly presents as a solitary lesion: The occurrence of multiple lesions is rare (1). GBM is most commonly occurs in individuals aged between 45 and 70 years old, with a worldwide incidence of 1-10\% (1-3).

Correspondence to: Mr. Sheng Zhang, Department of Radiology, The First Affiliated Hospital, College of Medicine, Zhejiang University, 79 Qingchun Road, Hangzhou, Zhejiang 310003, P.R. China

E-mail: zhangyly1@163.com

Key words: glioblastoma multiforme, magnetic resonance imaging, proliferation time
The most common clinical symptom of GBM is epilepsy (1). Magnetic resonance imaging (MRI) is the primary diagnostic tool for GBM (4). Tumors involving the corpus callosum, which grow bilaterally into occipital and temporal lobes, result in a butterfly pattern on MRI, termed 'butterfly glioma' (5). Definitive diagnosis is based on histopathological examination of intraoperatively removed tumors/tumor sections. Morphological diagnosis is based on criteria defined by the World Health Organization $(6,7)$. Surgical resection is recommended if feasible, followed by chemotherapy and radiotherapy, which is the standard treatment for GBM (8). GBM exhibits a highly unfavorable prognosis; The majority of patients only survive for 12-15 months following disease onset (9). Previous in vivo studies of GBM have focused on the etiology, pathology, clinical symptoms, imaging features, treatment and prognosis of the disease (1-3). In vitro studies of GBM cell proliferation have also been performed $(10,11)$, however, the mechanism of disease progression and its corresponding MRI features remain unclear. In this study, a case of rapidly progressing GBM is presented, and the findings of MRI, surgical and pathological examinations are discussed. Written informed consent was obtained from the patient.

\section{Case report}

In April 2010, a 60-year-old male patient with mental confusion was admitted to The First Affiliated Hospital (Hangzhou, China) after experiencing convulsions for 1 day. Physical examination revealed that the pupil size of both eyes was equal; however, the pupillary light reflex was slow. In addition, high muscular tension of the limbs was observed and positive bilateral Babinski signs were identified. Blood examinations revealed a leukocyte count of $12.0 \times 10^{9} / 1$ and a neutrophil granulocyte level of $92.6 \%$. Kidney function and blood gas composition were normal. MRI examination revealed abnormal signals from the temporal-occipital-parietal lobes and thus metastatic tumors were initially suspected and the possibility of a brain abscess was excluded (Fig. 1). The patient reported no history of disease, however, he had experienced head trauma with basal fracture 10 years previously. Serum tumor markers, including carcinoembryonic antigen, $\alpha$-fetoprotein, carbohydrate antigen 199 and 125, total prostate specific antigen and ferritin were normal. Furthermore, erythrocyte sedimentation rate was normal and serum was negative for antinuclear (cat. no. FA 1510-1003-1; 1:10; EUROIMMUN AG, Luebeck, germany) and antineutrophil 

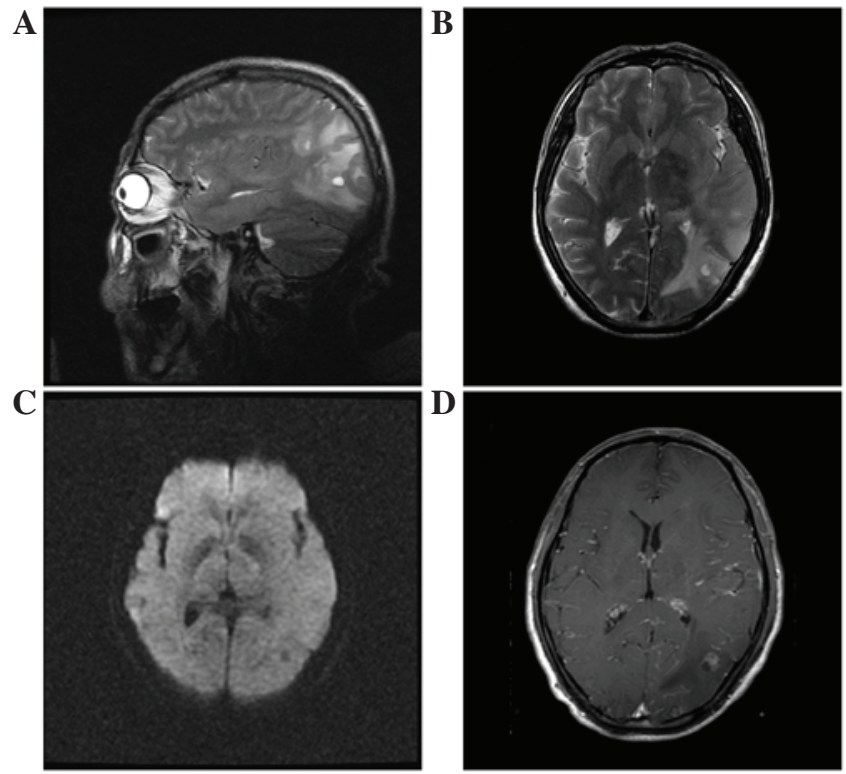

Figure 1. Magnetic resonance imaging performed following initial hospitalization. (A) Sagittal and (B) axial T2-weighted images revealed multiple round, long signals in the left temporal, parietal and occipital lobes. A cyst-solid lesion, $\sim 7 \mathrm{~mm}$ in diameter, with clear edges was identified. Furthermore, the inferior horn of the lateral ventricles was evidently compressed. (C) Axial diffusion-weighted images revealed homogenous signals (D) Enhanced axial T1-weighted images demonstrated marked enhancement of the cystic wall and the solid nodule.
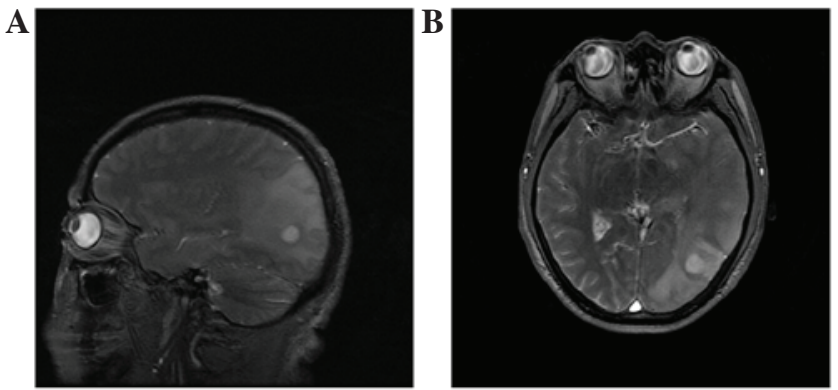

Figure 2. Magnetic resonance imaging performed 12 days after hospitalization. (A) Sagittal and (B) axial T2-weighted images revealed that lesion size had increased and the diameter of the maximal cyst-solid lesion increased to $13 \mathrm{~mm}$, with enlargement of the lesion cavity. The inner wall of the tumor was smooth.

cytoplasmic (cat. no. FA 1200-1003; 1:10; EUROIMMUN AG) antibodies.

Cerebrospinal fluid (CSF) pressure was $180 \mathrm{mmH}_{2} \mathrm{O}$ (normal range, $80-180 \mathrm{mmH}_{2} \mathrm{O}$ ) with transparency and no blood cells. Biochemical analysis of the CSFrevealed a protein concentration of $0.49 \mathrm{~g} / 1$ (normal range, $0.15-0.45 \mathrm{~g} / \mathrm{l}$ ) and carcinoembryonic antigen levels of $<0.50 \mathrm{ng} / \mathrm{ml}$ (normal range, $<0.573 \mathrm{ng} / \mathrm{ml}$ ). Glucose and chloride levels were within the normal ranges. Computed tomography (CT) images of the lung were normal. Electroencephalogram demonstrated a slow spike wave in the left hemisphere in addition to an epileptic lesion in the left central parietal lobe.

Subsequently, the patient was administered mannitol ( $125 \mathrm{ml}$, every $12 \mathrm{~h}$ for 26 days) to lower intracranial pressure, diazepam (10 mg, daily for 3 days), Tegretol ( $0.2 \mathrm{~g}$, twice daily for 26 days) to treat the symptoms of epilepsy and ceftriaxone
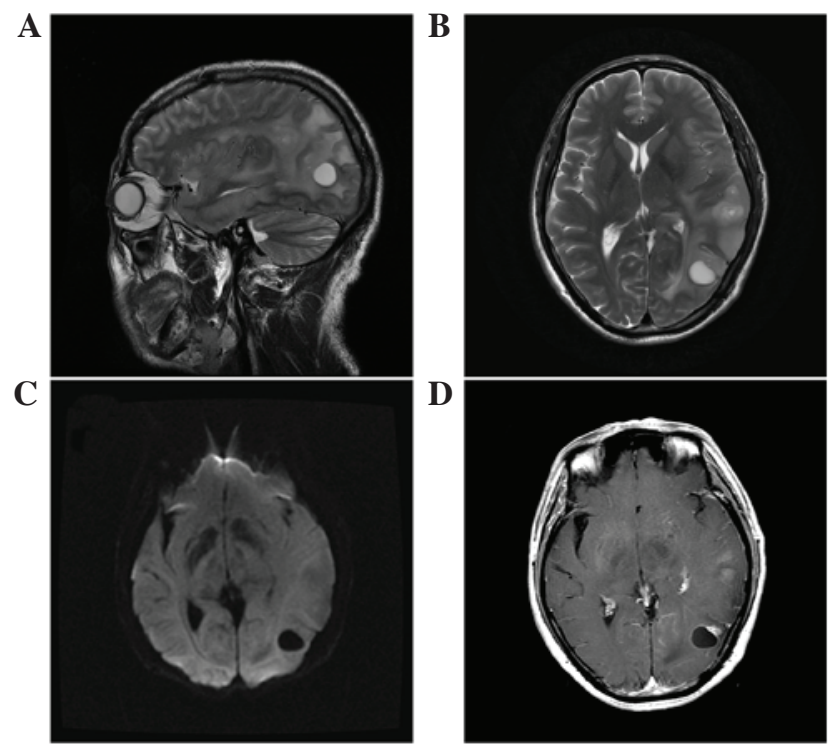

Figure 3. Magnetic resonance imaging performed 23 days after hospitalization. (A) Sagittal and (B) axial T2-weighted images revealed a larger lesion, $17 \mathrm{~mm}$ in diameter. The tumor exhibited clear edges and homogenous signals in the cavity in addition to peritumoral edema. (C) Axial diffusion-weighted images revealed homogenous signals. (D) Enhanced axial T1-weighted images revealed weak enhancement of the cystic wall in contrast to marked enhancement of the solid nodule.

A
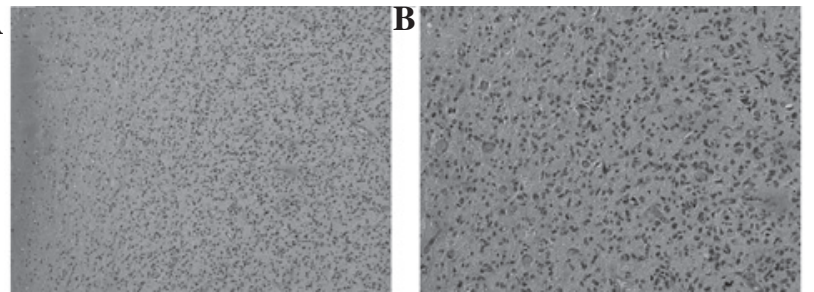

Figure 4. Histopathological findings. (A) Proliferating tumor cells and heterocysts were observed with abundant small vessels. Necrosis was observed on the left side (magnification, x10). (B) Proliferating tumor cells and heterocysts were observed with abundant small vessels (magnification, $\mathrm{x} 40$ ).
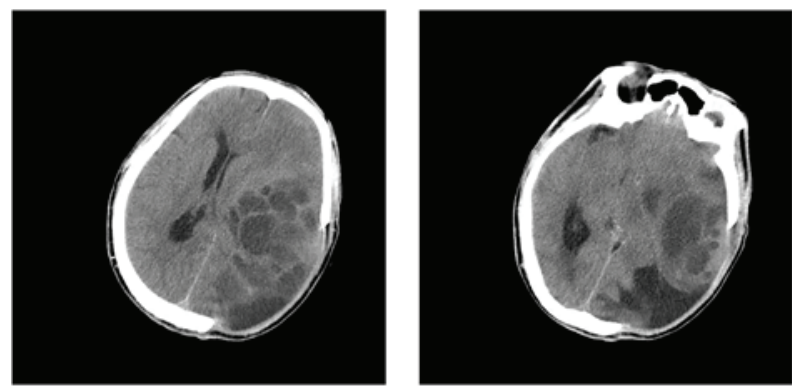

Figure 5. Computed tomography imaging performed 7 months after surgery revealing a multiloculated cystic lesion located at the left temporoparietal junction (multiple views). The position effect was evident, indicating tumor recurrence.

( $2.0 \mathrm{~g}$, daily for 9 days) for infection control. After treatment, the patient recovered and regained full consciousness with a normal body temperature and was able to eat and walk without the occurrence of seizures. MRI examination performed 12 days after hospitalization revealed multiple lesions between the left temporal and left occipital lobes, which had increased 
in size since the initial MRI examination, and thus parasitic infection could not be ruled out (Fig. 2). The spirometra mansoni test was positive. Therefore, the patient remained hospitalized to treat the infection. MRI examination 23 days after hospitalization revealed multiple lesions, which had markedly increased in size and number when compared with the previous MRI findings. Subsequently, GBM was suspected (Fig. 3) and resection of tumor in the left temporal occipital lobe was performed. Histopathological examination identified GBM [World Health Organization grade IV (12)] in the left temporal and parietal lobes (Fig. 4). No further treatment was administered. Seven months after surgery, the patient experienced a sudden headache and dysfunction of the extremities. CT of the head revealed GBM recurrence (Fig. 5). The patient was subsequently administered mannitol treatment, however he succumbed to the disease in January 2011.

\section{Discussion}

GBM [World Health Organization stage IV (12)] is one of the most malignant tumors worldwide. The first case of GBM was reported by Bradley in 1880 (13). At present, the incidence rate of GBM is $1-10 \%(1,13)$. Due to the high grade of malignancy, GBM exhibits a highly unfavorable prognosis; some patients succumbed within 3 months of disease onset (14). GBM exhibits no specific clinical, imaging or histological characteristics. Depending on the localization and progression of GBM, the clinical symptoms may include headache, ataxia, dizziness, visual disturbances and frequent syncope $(15,16)$. The occurrence of seizures in patients who have not been previously diagnosed with epilepsy may also be an indication for neuroimaging due to suspected GBM (2). Due to the lack of specific symptoms, GBM is often misdiagnosed as an infection, inflammatory process or circulatory and immunological diseases (15). The patient in the present case was hospitalized due to the occurrence of seizures.

GBM tumors develop as a result of infiltrative growth via nerve fiber pathways or metastatic spread via CSF, therefore satellite lesions form in adjacent areas of the brain $(1,13)$. Multiple lesions were identified in the present case and we hypothesize that the lesions located near the sulci may have been caused by local spread. GBM develops as a result of infiltrative growth, which increases intracranial pressure and subsequently may lead to hydrocephaly (3). The inferior horn of the lateral ventricles of the left hemisphere was evidently compressed in the present case. The aggravation of clinical symptoms may be associated with the rapid growth of glioblastoma, which damages local tissue structure and increases the position effect causing impaired brain function.

MRI is the primary diagnostic tool for GBM. Simpson et al (8) evaluated the MRI findings of 645 GBM patients and reported that in 38, 56 and $6 \%$ of patients, the tumor diameter at diagnosis was $<5,5-10$ and $>10 \mathrm{~cm}$, respectively. The high proliferation rate of GBM causes rapid tumor growth, and a previous study reported that the tumor cell doubling time for GBM ranges between 2 days and several weeks (10). It is hypothesized that tumor cell doubling time may predict survival time $(10,14)$. The median survival time following diagnosis for patients with GBM is 9.8 days (10). To the best of our knowledge, no in vivo studies have investigated the imaging features that are associated with GBM progression. In the present case, the initial MRI examination indicated that the maximum diameter of the lesion on the left temporal-occipital lobe was $\sim 7 \mathrm{~mm}$ with cystic and enhanced solid sections. MRI performed 12 days after hospitalization revealed that the lesion had increased to $13 \mathrm{~mm}$ in diameter. The lesion exhibited revealed homogenous low signals on diffusion-weighted images, however, the peri-tumor edema range remained unchanged. MRI performed 23 days after hospitalization revealed that the diameter of the lesion had increased to $17 \mathrm{~mm}$ in the cystic sections and the solid sections exhibited enhancement. The rapid increase in lesion number and size demonstrated by MRI imaging in the present case was in accordance with a previous in vitro study of GBM cell culture (10). Thus, the rapid morphological progression of cystic lesion sections may indicate GBM. In the meanwhile, the enlarged GBM is mainly from the cystic parts rather than solid parts might attribute to the necrotic features of GBM (17). In the present study, diagnosis of GBM was uncertain in the early stages due to the positive results obtained from the spirometra mansoni test. However, histopathological examination following surgery confirmed the diagnosis of GBM. In cases where a tumor is suspected and resection procedures are limited, fine needle biopsy is recommended to minimize trauma (18). The present case revealed that GBM may progress rapidly with a doubling time of 10 days and multiple cystic alterations. If diagnosis of GBM is unclear, early biopsy is recommended.

\section{References}

1. Salvati M, Caroli E, Orlando ER, Frati A, Artizzu S and Ferrante L: Multicentric glioma: Our experience in 25 patients and critical review of the literature. Neurosurg Rev 26: 275-279, 2003.

2. Sanli AM, Turkoglu E, Dolgun H and Sekerci Z: Unusual manifestations of primary Glioblastoma Multiforme: A report of three cases. Surg Neurol Int 1: 87, 2010.

3. de Castro-Costa CM, de Araújo RW, de Arruda MA, de Araújo PM and de Figueiredo EG: Increased intracranial pressure in a case of spinal cervical glioblastoma multiforme. Analysis of these two rare conditions. Arq Neuropsiquiatr 52: 64-68, 1994.

4. Ulutin C, Fayda M, Aksu G, Cetinayak O, Kuzhan O, Ors F and Beyzadeoglu M: Primary glioblastoma multiforme in younger patients: A single-instruction experience. Tumori 92: 407-411, 2006.

5. Agrawal A: Butterfly glioma of the corpus callosum. J Cancer Res Ther 5: 43-45, 2009.

6. Louis DN, Ohgaki H, Wiestler OD and Cavenee WK (eds): WHO Classification of Tumours of the Central Nervous System. Vol 1. 4th edition. IARC Press, Lyon, 2007.

7. Zülch KJ (ed): Histological Typing of Tumours of the Central Nervous System. World Health Organization, Geneva, 1979.

8. Simpson JR, Horton J, Scott C, Curran WJ, Rubin P, Fischbach J, Isaacson S, Rotman M, Asbell SO, Nelson JS, et al: Influence of location and extent of surgical resection on survival of patients with glioblastoma multiforme: Results of three consecutive Radiation Therapy Oncology Group (RTOG) clinical trials. Int J Radiat Oncol Biol Phys 26: 239-244, 1993.

9. Wen PY and Kesari S: Malignant gliomas in adults. N Engl J Med 359: 492-507, 2008.

10. Furneaux CE, Marshall ES, Yeoh K, Monteith SJ, Mews PJ, Sansur CA, Oskouian RJ, Sharples KJ and Baguley BC: Cell cycle times of short-term cultures of brain cancers as predictors of survival. Br J Cancer 99: 1678-1683, 2008.

11. Qu J, Rizak JD, Fan Y, Guo X, Li J, Huma T and Ma Y: Establishment and partial characterization of a human tumor cell line, GBM-HSF, from a glioblastoma multiforme. Human Cell 27: 129-136, 2014. 
12. Fuller GN and Scheithauer BW: The 2007 Revised World Health Organization (WHO) Classification of Tumours of the Central Nervous System: newly codified entities. Brain Pathol 17: 304-307, 2007.

13. Bradley WL: Case of gliosarcomatous tumors of the brain. Proc Con Med Soc 2: 39-41, 1880

14. Cvetkoivč-Dožič D, Skender-Gazibara M and Dožič S: Morphological and molecular features of diffuse infiltrating astrocytoma. Arch Oncol 12: 38-39, 2004

15. Lakhan SE and Harle L: Difficult diagnosis of brainstem glioblastoma multiforme in a woman: A case report and review of the literature. J Med Case Rep 3: 87, 2009.
16. Levine SA, McKeever PE and Greenberg HS: Primary cerebellar glioblastoma multiforme. J Neurooncol 5: 231-236, 1987.

17. Kleihues P, Burger PC, Collins VP and Cavenee WK (eds): World Health Organization Classification of Tumours. Pathology and Genetics of Tumours of the Nervous System. IARC Press, Lyon, 2000.

18. Katsetos CD, Dráberová E, Legido A and Dráber P: Tubulin targets in the pathobiology and therapy of glioblastoma multiforme. II. gamma-Tubulin. J Cell Physiol 221: 514-520, 2009. 